\title{
ENFERMAGEM BASEADA EM EVIDÊNCIA: CARACTERIZAÇÃO DOS ESTUDOS NO BRASIL
}

Karilena Karlla Amorim Pedrosa', Isabelle Christine Marinho Oliveira1', Alexsandra Rodrigues Feijão², Regimar Carla Machado ${ }^{3}$

${ }^{1}$ Enfermeira. Mestranda em Enfermagem. Universidade Federal do Rio Grande do Norte. Natal, RN, Brasil.

Enfermeira. Doutora em Enfermagem. Docente do Departamento de Enfermagem da Universidade Federal do Rio Grande do Norte. Natal, RN, Brasil.

${ }^{3}$ Enfermeira. Pós-Doutorado em Cirurgia Translacional. Docente do Departamento de Enfermagem da Universidade Federal de São Carlos. São Carlos, SP, Brasil.

RESUMO: Revisão integrativa que objetivou caracterizar estudos brasileiros sobre prática de enfermagem baseada em evidências. A busca foi realizada no período de junho a setembro de 2014, na Literatura Latino-Americana e do Caribe em Ciências da Saúde, Scientific Electronic Library Online, e National Library of Medicine National Institutes of Health. Foram selecionados 21 artigos, todos publicados em periódicos nacionais, no período de 2003 a 2014. Os resultados apontam para um maior número de revisões, com nível de evidência 5, em detrimento dos demais tipos de estudo encontrados. Com destaque, têm-se as revisões integrativas e sistemáticas direcionadas para a prática assistencial e de ensino, desenvolvidas na região sudeste, por enfermeiros com atuação no ensino com titulação máxima de doutor. Ressalta-se a necessidade de capacitação do enfermeiro assistencial na busca, desenvolvimento e utilização de pesquisas na prática, a fim de transpor a dicotomia entre a pesquisa e o cuidar. DESCRITORES: Prática clínica baseada em evidências; Enfermagem baseada em evidências; Pesquisa em enfermagem; Enfermagem.

\section{EVIDENCE-BASED NURSING: CHARACTERISTICS OF STUDIES IN BRAZIL}

\begin{abstract}
The purpose of this integrative review was to characterize Brazilian studies on the practice of evidencebased nursing practice. The literature search was carried out between June and September 2014 in the Latin American and Caribbean Health Science Literature Database, the Scientific Electronic Library Online, and the National Library of Medicine National Institutes of Health. Twenty-one articles were selected, all published in Brazilian journals between 2003 and 2004. The results indicate a greater number of level 5 reviews in comparison to other types of studies. Of these, most were integrative and systematic reviews directed at healthcare and teaching practices, developed in the Southeast of Brazil, by nursing professors who have a doctoral degree as their highest qualification. In conclusion, nurses in the healthcare context must be trained to search for, develop and use research in practical contexts in order to overcome the dichotomy between research and healthcare provision.
\end{abstract}

DESCRIPTORS: Evidence-based practice; Evidence-based nursing; Nursing research; Nursing.

\section{ENFERMERÍA BASADA EN EVIDENCIA: CARACTERIZACIÓN DE LOS ESTUDIOS EN BRASIL}

RESUMEN: Revisión integrativa cuya finalidad fue caracterizar estudios brasileños sobre práctica de enfermería basada en evidencias. La búsqueda fue realizada en el periodo de junio a septiembre de 2014, en la Literatura Latinoamericana y del Caribe en Ciencias de la Salud, Scientific Electronic Library Online, y National Library of Medicine National Institutes of Health. Fueron seleccionados 21 artículos, todos publicados en periódicos nacionales, en el periodo de 2003 a 2014. Los resultados apuntan para un mayor número de revisiones, con nivel de evidencia 5, en detrimento de los demás tipos de estudio investigados. Se destacan revisiones integrativas y sistemáticas direccionadas para la práctica asistencial y de enseñanza, desarrolladas en la región sudeste, por enfermeros con actuación en la enseñanza con titulación máxima de doctor. Se destaca la necesidad de capacitación del enfermero asistencial en la búsqueda, desarrollo y utilización de investigaciones en la práctica, a fin de transponer la dicotomía entre la investigación y el cuidar. DESCRIPTORES: Práctica clínica basada en evidencias; Enfermería basada en evidencias; Investigación en enfermería; Enfermería. 


\section{INTRODUÇÃO}

O uso de evidências científicas em enfermagem tem como pressuposto a realização de estudos que gerem práticas inovadoras, com vista a sustentar as ações e as relações do profissional enfermeiro no sistema de saúde, bem como demonstrar o impacto de tais evidências na prática ${ }^{(1)}$.

A Prática Baseada em Evidências (PBE) como forma segura e organizada de estabelecer condutas profissionais, possui seu enfoque na solução de problemas alicerçado nas melhores evidências científicas ${ }^{(2-3)}$. Envolve a definição de um problema, a busca e avaliação crítica das evidências disponíveis para melhor tomada de decisão, implementação, e avaliação dos resultados obtidos, assim como a integração desses elementos com a competência clínica do profissional de saúde e as preferências do paciente $^{(4-5)}$.

Ao fundamentar a PBE, a evidência científica tem o poder de agregar ciência, visto que representa a confiabilidade em determinado conhecimento nos preceitos científicos ${ }^{(6-7)}$. Portanto, é oriunda da pesquisa clínica relevante, focada no paciente, para aprimoramento das medidas de diagnóstico, indicadores de prognóstico e tratamento, reabilitação e prevenção ${ }^{(5)}$.

Assim, a complexidade das informações necessárias à área da saúde e o tempo limitado dos profissionais na busca de resultados oriundos de pesquisas, têm ressaltado a importância das revisões neste cenário, principalmente das revisões sistemáticas, como recurso nas buscas das evidências necessárias ${ }^{(8)}$.

Embora a PBE sob o método de revisão sistemática (RS) de ensaios clínicos controlados e aleatórios seja considerada o melhor na análise e síntese de pesquisas clínicas, diversas revisões integrativas têm sido realizadas. Estas contribuem para $\mathrm{O}$ aprofundamento do conhecimento do tema investigado, de modo a promover a qualidade dos serviços de saúde e a diminuição dos custos operacionais ${ }^{(3,9-10)}$.

As mudanças geradas por meio da utilização da prática de enfermagem baseada em evidência são relevantes para um maior desenvolvimento profissional. No entanto, são poucos os enfermeiros que atuam em ambientes que centram suas ações em evidências científicas. Determinadas práticas do cuidar ainda acontecem por meio do conhecimento empírico, o que torna a prática incipiente, em face dos avanços tecnológicos e aquisições ao processo de cuidar.

Com o intuito de conhecer melhor as pesquisas sobre PBE realizados por enfermeiros no Brasil, e oferecer subsídios que proporcionem reflexões sobre o uso da PBE na Enfermagem, esse estudo teve como questão norteadora: Quais as características de estudos referentes à prática de enfermagem baseada em evidência, publicada por pesquisadores brasileiros?

Diante do exposto, procurando contribuir para a melhoria dos estudos em enfermagem e maior divulgação de conhecimentos científicos, propôsse a realização deste estudo com o objetivo de caracterizar os estudos brasileiros sobre prática de enfermagem baseada em evidência.

\section{MÉTODO}

Trata-se de revisão integrativa $(\mathrm{RI})$ da literatura, realizada em seis fases: elaboração da pergunta norteadora, seguida pela busca na literatura, coleta de dados, análise crítica dos estudos incluídos, discussão dos resultados e apresentação da revisão ${ }^{(11)}$.

Com base na questão norteadora, a busca dos artigos foi realizada por pares, no período de junho a setembro de 2014, na Literatura LatinoAmericana e do Caribe em Ciências da Saúde (LILACS), Scientific Electronic Library Online (SciELO) utilizando de forma não-controlada os Descritores em Ciências da Saúde (DeCS): prática clínica baseada em evidências, enfermagem baseada em evidências, medicina baseada em evidências, pesquisa em enfermagem, enfermagem. Enquanto na National Library of Medicine National Institutes of Health (PubMed) foram empregados de forma controlada os descritores Medical Subject Headings (MeSH): Evidence-Based Practice, Evidence-Based Nursing, Evidence-Based Medicine, Nursing Research, Nursing. A interação desses descritores foi realizada pelo operador booleano AND.

Para a seleção dos estudos foram utilizados, como critérios de inclusão, artigos completos disponíveis que abordem estudos brasileiros sobre enfermagem baseada em evidência e como critérios de exclusão os editoriais e cartas ao editor.

Com estratégia de busca, foram encontrados 423 artigos completos. Para esta revisão, foram pré-selecionados 31 artigos, com base na análise do título e resumo. Os artigos duplicados foram computados apenas uma vez. Em seguida, os 
artigos que apresentavam conformidade com os critérios de inclusão dessa revisão foram avaliados na íntegra. Resultando em uma amostra de 21 estudos, como pode ser observado na Tabela 1.

Tabela 1 - Estratégia de busca eletrônica nas bases de dados e bibliotecas virtuais. Natal, RN, Brasil, 2014

\begin{tabular}{|c|c|c|c|c|}
\hline \multirow{2}{*}{\multicolumn{2}{|c|}{ CRUZAMENTOS }} & \multicolumn{3}{|c|}{$\begin{array}{c}\text { BASES DE DADOS E } \\
\text { BIBLIOTECAS VIRTUAIS }\end{array}$} \\
\hline & & LILACS & SciELO & PubMed \\
\hline 1. & $\begin{array}{l}\text { Prática clínica } \\
\text { baseada em } \\
\text { evidências AND } \\
\text { Enfermagem baseada } \\
\text { em evidências }\end{array}$ & 1 & 5 & 2 \\
\hline 2. & $\begin{array}{l}\text { Enfermagem baseada } \\
\text { em evidências } \\
\text { AND Pesquisa em } \\
\text { enfermagem }\end{array}$ & 2 & 2 & 0 \\
\hline 3. & $\begin{array}{l}\text { Prática clínica } \\
\text { baseada em } \\
\text { evidências AND } \\
\text { Pesquisa em } \\
\text { enfermagem }\end{array}$ & 0 & 2 & 0 \\
\hline 4. & $\begin{array}{l}\text { Medicina baseada } \\
\text { em evidências AND } \\
\text { Enfermagem }\end{array}$ & 15 & 0 & 4 \\
\hline \multicolumn{2}{|c|}{$\begin{array}{l}\text { Total de artigos pré- } \\
\text { selecionados }\end{array}$} & 18 & 7 & 6 \\
\hline \multicolumn{2}{|c|}{ Amostra final } & 13 & 5 & 4 \\
\hline
\end{tabular}

Para categorização dos dados, foi utilizado o instrumento $^{(12)}$ adaptado pelas pesquisadoras, contendo dados referentes à identificação do artigo (título, periódico, ano de publicação, local de busca, local do estudo e palavras-chave indicadas); e identificação da pesquisa (objetivo, tipo de estudo, nível de evidência, áreas de atenção em saúde, de conceitos/modelos teóricos, resultados e conclusões); e identificação dos pesquisadores (profissão, área de atuação e titulação máxima).

A avaliação dos estudos quanto ao nível de evidência (NE) seguiu a Oxford Centre EvidenceBased Medicine $^{(13)}$, como mostra o Quadro 1.

A apresentação dos resultados foi feita por meio de quadro comparativo e de forma descritiva. Para a caracterização dos estudos, utilizou-se frequência absoluta e relativa, com o intuito de viabilizar melhor análise dos achados e apresentação das evidências da revisão integrativa.
Quadro 1 - Níveis de evidência por tipo de estudo. Natal, RN, Brasil, 2014

\begin{tabular}{|c|c|}
\hline NE* & TIPOS DE ESTUDO \\
\hline $1 \mathrm{~A}$ & $\begin{array}{l}\text { Revisões sistemáticas e metanálises de ensaios } \\
\text { clínicos comparáveis. Estudos controlados } \\
\text { randomizados bem delineados com desfecho } \\
\text { clínico relevante. }\end{array}$ \\
\hline $1 \mathrm{~B}$ & $\begin{array}{l}\text { Estudos controlados randomizados com } \\
\text { estreito intervalo de confiança. }\end{array}$ \\
\hline $1 \mathrm{C}$ & $\begin{array}{l}\text { Resultados do tipo "tudo ou nada". Estudo de } \\
\text { série de casos controlados. }\end{array}$ \\
\hline $2 \mathrm{~A}$ & $\begin{array}{l}\text { Revisão sistemática homogênea de estudos de } \\
\text { coorte (com grupos de comparação e controle } \\
\text { de variáveis). }\end{array}$ \\
\hline $2 \mathrm{~B}$ & $\begin{array}{l}\text { Estudo de coorte com pobre qualidade } \\
\text { de randomização, controle ou sem } \\
\text { acompanhamento longo, estudo de coorte } \\
\text { transversal. }\end{array}$ \\
\hline $2 \mathrm{C}$ & $\begin{array}{l}\text { Resultados de pesquisas (observação de } \\
\text { resultados terapêuticos ou evolução clínica). }\end{array}$ \\
\hline $3 \mathrm{~A}$ & $\begin{array}{l}\text { Revisão sistemática homogênea de estudos de } \\
\text { caso com grupo-controle. }\end{array}$ \\
\hline 3B & Estudos de caso com grupo-controle. \\
\hline 4 & $\begin{array}{l}\text { Relatos de caso e série sem definição de caso- } \\
\text { controle. }\end{array}$ \\
\hline 5 & 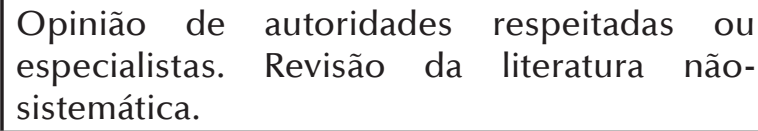 \\
\hline
\end{tabular}

\section{RESULTADOS}

A amostra desta revisão foi composta por 21 estudos. Todos os artigos foram codificados para melhor identificação e relação dos achados. O Quadro 2 expõe as características dos estudos segundo autores, referência, ano de publicação, periódico, local de busca.

A seguir, o Quadro 3 caracteriza os artigos segundo o tipo de estudo, nível de evidência, áreas de atenção da enfermagem e tema de cada estudo selecionado.

Com relação ao tipo de estudo, 12 (57,1\%) são artigos de revisão, sendo seis $(21,6 \%)$ revisões integrativas $(\mathrm{RI})$, duas $(9,5 \%)$ revisões sistemáticas (RS), das quais duas (9,5\%) utilizaram os ensaios clínicos randomizados e uma $(4,7 \%)$ metassíntese. Os demais são estudos de reflexão $(9,5 \%)$, atualização $(9,5 \%)$, descritivo $(4,7 \%)$, ensaio $(4,7 \%)$, metodológico $(4,7 \%)$, convergente assistencial $(4,7 \%)$ e relato de caso $(4,7 \%)$. Desses estudos, 11 (52,4\%) estão direcionados para uma intervenção de enfermagem na prática e no ensino com base nas evidências, e 10 
Quadro 2 - Caracterização dos estudos segundo código A1 a A21, autores, referência, ano de publicação, periódico, e local de busca. Natal, RN, Brasil, 2014

\begin{tabular}{|c|c|c|c|c|}
\hline CÓDIGO & AUTORES (Referência) & ANO & PERIÓDICO & $\begin{array}{l}\text { LOCAL DE } \\
\text { BUSCA } \\
\end{array}$ \\
\hline A1 & Azevedo, Barbosa, Cassiani ${ }^{(14)}$ & 2012 & Acta Paulista de Enfermagem & LILACS \\
\hline $\mathrm{A} 2$ & Silva, Chaves, Carvalho, Iunes ${ }^{(15)}$ & 2013 & Acta Paulista de Enfermagem & LILACS \\
\hline A3 & $\begin{array}{l}\text { Pedrolo, Danski, Mingorance, } \\
\text { Lazzari, Méier, Crozeta }{ }^{(5)}\end{array}$ & 2009 & Cogitare Enfermagem & LILACS \\
\hline A4 & Silva, Galvão, Pereira, Silva ${ }^{(16)}$ & 2013 & $\begin{array}{l}\text { Revista da Sociedade Brasileira de } \\
\text { Medicina Tropical }\end{array}$ & LILACS \\
\hline A5 & $\begin{array}{l}\text { Rabelo, Aliti, Domingues, Assis, Saffi, } \\
\text { Linhares, et } a /^{(17)}\end{array}$ & 2007 & Clinical \& Biomedical Research & LILACS \\
\hline A6 & Karino, Felli ${ }^{(18)}$ & 2012 & Ciência, Cuidado e Saúde & LILACS \\
\hline A7 & $\begin{array}{l}\text { Vasconcelos, Damasceno, Lima, } \\
\text { Pinheiro }^{(9)}\end{array}$ & 2011 & $\begin{array}{l}\text { Revista Latino-Americana de } \\
\text { Enfermagem }\end{array}$ & LILACS \\
\hline A8 & Galvão, Sawada, Mendes ${ }^{(19)}$ & 2003 & $\begin{array}{l}\text { Revista da Escola de Enfermagem da } \\
\text { USP }\end{array}$ & LILACS \\
\hline A9 & Galvão, Sawada(10) & 2003 & Revista Brasileira de Enfermagem & LILACS \\
\hline A10 & Santos, Pimenta, Nobre ${ }^{(6)}$ & 2007 & $\begin{array}{l}\text { Revista Latino-Americana de } \\
\text { Enfermagem }\end{array}$ & LILACS \\
\hline A11 & Cruz, Pimenta $^{(7)}$ & 2005 & $\begin{array}{l}\text { Revista Latino-Americana } \\
\text { Enfermagem }\end{array}$ & LILACS \\
\hline A12 & Galvão, Sawada, Trevizan ${ }^{(8)}$ & 2004 & $\begin{array}{l}\text { Revista Latino-Americana de } \\
\text { Enfermagem }\end{array}$ & SciELO \\
\hline A13 & Domenico, Ide ${ }^{(20)}$ & 2003 & $\begin{array}{l}\text { Revista Latino-Americana } \\
\text { Enfermagem }\end{array}$ & SCiELO \\
\hline A14 & Santos, Carvalho ${ }^{(21)}$ & 2012 & Revista Brasileira de Enfermagem & SciELO \\
\hline A15 & Mendes, Galvão(22) & 2008 & $\begin{array}{l}\text { Revista Latino-Americana de } \\
\text { Enfermagem }\end{array}$ & SciELO \\
\hline A16 & Silva, Nascimento, Salles ${ }^{(23)}$ & 2012 & Texto \& Contexto Enfermagem & SCIELO \\
\hline A17 & $\begin{array}{l}\text { Soares, Hoga, Peduzzi, Sangaleti, } \\
\text { Yonekura, Silva }{ }^{(24)}\end{array}$ & 2014 & $\begin{array}{l}\text { Revista da Escola de Enfermagem da } \\
\text { USP }\end{array}$ & SCiELO \\
\hline A18 & $\begin{array}{l}\text { Júnior, Santos, Moura, Melo, } \\
\text { Monteiro }\end{array}$ & 2011 & Revista Brasileira de Enfermagem & PubMed \\
\hline A19 & $\begin{array}{l}\text { Lacerda, Nunes, Batista, Egry, } \\
\text { Graziano, Angelo, et a/ }{ }^{(3)}\end{array}$ & 2012 & $\begin{array}{l}\text { Revista da Escola de Enfermagem da } \\
\text { USP }\end{array}$ & PubMed \\
\hline A20 & Toma, Rea ${ }^{(26)}$ & 2008 & Cadernos de Saúde Pública & PubMed \\
\hline A21 & Oliveira, Camacho, Souza ${ }^{(27)}$ & 2005 & Cadernos de Saúde Pública & PubMed \\
\hline
\end{tabular}

$(47,6 \%)$ voltadas para metodologia de pesquisa, ressaltando a importância do método para prática do profissional da enfermagem, com a PBE como referencial teórico.

Dos artigos selecionados, todos estão publicados em periódicos nacionais, sendo que dois pertencem a uma revista médica, e os demais estão publicados em revistas de enfermagem, com destaque para revista Latino Americana de Enfermagem com seis publicações. Quanto ao recorte temporal de publicação, observou-se que os estudos são recentes na literatura com o intervalo entre 2003 e 2014, pois datam dos últimos 10 anos, com uma maior concentração nos últimos cinco anos. Com apenas um artigo na língua inglesa(15), os demais em português.

Quanto ao local dos estudos, 15 (71,4\%) artigos são provenientes da região Sudeste, três $(14,2 \%)$ da região Sul, dois $(9,6 \%)$ da Nordeste e um $(4,8 \%)$ do Centro-Oeste brasileiro. Sendo realizados com maior recorrência nos estados de São Paulo e Rio de Janeiro, regiões de maior concentração de universidades e de maior divulgação do conhecimento científico por meio de periódicos.

Os descritores identificados nos estudos 
Quadro 3 - Caracterização dos estudos código A1 a A21 segundo tipo de estudo, nível de evidência, áreas de atenção da enfermagem e tema do estudo. Natal, RN, Brasil, 2014

\begin{tabular}{|c|c|c|c|c|}
\hline CÓDIGO & $\begin{array}{l}\text { TIPO DE } \\
\text { ESTUDO }\end{array}$ & NE & $\begin{array}{l}\text { ÁREAS DE } \\
\text { ATENÇÃO }\end{array}$ & TEMA DO ESTUDO \\
\hline A1 & $\begin{array}{l}\text { Revisão } \\
\text { integrativa }\end{array}$ & 5 & Oncologia & $\begin{array}{l}\text { Uso de antibióticos por via subcutânea em pacientes com } \\
\text { difícil acesso venoso em cuidados paliativos }\end{array}$ \\
\hline A2 & $\begin{array}{l}\text { Estudo } \\
\text { metodológico }\end{array}$ & 5 & Dermatologia & Avaliação dos pés de portadores de diabetes mellitus \\
\hline A3 & Reflexão & 5 & Pesquisa & $\begin{array}{l}\text { Prática Baseada em Evidências na prática profissional do } \\
\text { enfermeiro }\end{array}$ \\
\hline A4 & $\begin{array}{l}\text { Revisão com } \\
\text { ensaios clínicos } \\
\text { e estudos } \\
\text { observacionais }\end{array}$ & 1B & Dermatologia & $\begin{array}{l}\text { Tratamento da leishmaniose tegumentar americana em } \\
\text { populações especiais }\end{array}$ \\
\hline A5 & \begin{tabular}{|l|} 
Revisão com \\
ensaios clínicos \\
randomizados \\
\end{tabular} & 1B & Cardiologia & $\begin{array}{l}\text { Estratégias de educação e acompanhamento a pacientes } \\
\text { com insuficiência cardíaca }\end{array}$ \\
\hline A6 & Revisão & 5 & Pesquisa & $\begin{array}{l}\text { Contribuições do Instituto Joanna Briggs para a enfermagem } \\
\text { baseada em evidências }\end{array}$ \\
\hline A7 & $\begin{array}{l}\text { Revisão } \\
\text { integrativa }\end{array}$ & 5 & $\begin{array}{l}\text { Saúde da } \\
\text { mulher }\end{array}$ & $\begin{array}{l}\text { Intervenções de enfermagem utilizadas para detecção } \\
\text { precoce do câncer cérvico-uterino }\end{array}$ \\
\hline A8 & Atualização & 5 & Pesquisa & $\begin{array}{l}\text { A implementação da Prática baseada em evidências na } \\
\text { enfermagem }\end{array}$ \\
\hline A9 & Reflexão & 5 & Pesquisa & $\begin{array}{l}\text { A implementação da Prática baseada em evidências na } \\
\text { enfermagem }\end{array}$ \\
\hline A10 & Atualização & 5 & Pesquisa & Construção da pergunta de pesquisa na busca de evidências \\
\hline A11 & Revisão & 5 & Pesquisa & $\begin{array}{l}\text { Prática baseada em evidência (PBE) às decisões diagnósticas } \\
\text { no cuidado de enfermagem }\end{array}$ \\
\hline A12 & Revisão & 5 & Pesquisa & Revisão sistemática \\
\hline A13 & Relato de caso & 4 & Pesquisa & Enfermagem baseada em evidências \\
\hline A14 & $\begin{array}{l}\text { Revisão } \\
\text { integrativa com } \\
\text { ensaios clínicos } \\
\text { randomizados }\end{array}$ & 5 & Ortopedia & $\begin{array}{l}\text { Intervenções de enfermagem para o cuidado de pacientes } \\
\text { com artrite }\end{array}$ \\
\hline A15 & $\begin{array}{l}\text { Revisão } \\
\text { integrativa }\end{array}$ & 5 & Transplantes & $\begin{array}{l}\text { Cuidado de enfermagem prestado ao paciente adulto } \\
\text { submetido ao transplante de fígado }\end{array}$ \\
\hline A16 & $\begin{array}{l}\text { Estudo } \\
\text { convergente- } \\
\text { assistencial }\end{array}$ & $2 \mathrm{C}$ & $\begin{array}{l}\text { Cuidados } \\
\text { críticos }\end{array}$ & Prevenção da pneumonia associada à ventilação mecânica \\
\hline A17 & \begin{tabular}{|l} 
Revisão \\
integrativa \\
\end{tabular} & 5 & Pesquisa & Revisão integrativa \\
\hline A18 & \begin{tabular}{|l} 
Revisão \\
sistemática e \\
Metassíntese \\
\end{tabular} & $3 \mathrm{~A}$ & Terminalidade & Processo de morte e morrer \\
\hline A19 & $\begin{array}{l}\text { Revisão } \\
\text { integrativa }\end{array}$ & 5 & Pesquisa & $\begin{array}{l}\text { Práticas baseadas em evidências (PBE) acerca da prevenção } \\
\text { em saúde humana }\end{array}$ \\
\hline A20 & \begin{tabular}{|l|} 
Ensaio com \\
revisões \\
sistemáticas \\
\end{tabular} & 5 & $\begin{array}{l}\text { Saúde da } \\
\text { mulher e da } \\
\text { criança }\end{array}$ & Benefícios do aleitamento materno para a criança e mulher \\
\hline A21 & $\begin{array}{l}\text { Revisão } \\
\text { sistemática }\end{array}$ & $3 \mathrm{~A}$ & $\begin{array}{l}\text { Saúde da } \\
\text { mulher e da } \\
\text { criança }\end{array}$ & $\begin{array}{l}\text { Promoção, proteção e apoio à amamentação na atenção } \\
\text { primária }\end{array}$ \\
\hline
\end{tabular}


incluíram o total de 71 termos, presentes no DeCS. Dentre eles, podem ser citados os de maior recorrência: Prática clínica baseada em evidências, Enfermagem baseada em evidência se Medicina baseada em evidências (15,5\%), Pesquisa em enfermagem (11,2\%), Cuidados de Enfermagem (11,2\%), Revisão, Revisão Sistemática e Metanálise, (8,4\%), Enfermagem(7\%), e Tomada de Decisões $(2,8 \%)$.

Os autores dos estudos totalizaram 76 pesquisadores. Destes, $82,2 \%$ representavam enfermeiros e $17,8 \%$ de outras profissões, como medicina, farmácia, fisioterapia e biblioteconomia. Quando explorada a área de atuação dos enfermeiros, prevaleceu, em $69,3 \%$, a docência do ensino superior, seguida por $16,9 \%$ da assistência e 13,8\% de alunos da pós-graduação e graduação. Quanto aos enfermeiros-autores, $61,5 \%$ detinham o título de doutor, $17 \%$ o de mestre, $1,5 \%$ o de especialista, os demais $20 \%$ eram alunos da pósgraduação e graduação.

\section{DISCUSSÃO}

O processo do cuidar é beneficiado com os avanços tecnológicos atuais, destarte é necessário compreender o impacto que estes avanços apresentam nesse processo. Para isso, surge a necessidade de validar conhecimentos e produzir evidências que subsidiem sua aplicação. Nesse contexto, a PBE representa a forma como este impacto será compreendido(5).

No Brasil, embora esse movimento ainda seja pouco utilizado na enfermagem, observase um crescimento de pesquisas nessa área. O desenvolvimento crescente de pesquisas com este enfoque na área de enfermagem tem resultado, nos últimos anos, em estudos tendo a PBE como referencial teórico ou abordando as suas estratégias metodológicas para pesquisas de qualidade baseada em evidência, ressaltando a importância do método para prática do profissional da enfermagem ${ }^{(5)}$, como mostram os resultados desses estudo.

Dependendo da área de prática assistencial e seus enfoques, as metodologias passíveis de aplicação são empregadas. A RI é utilizada na PBE e apresenta uma tendência na enfermagem, como observado nos resultados deste estudo, pois houve elevada frequência de artigos desta natureza. Ressalta-se que este aspecto pode estar relacionado ao potencial desta metodologia em sintetizar achados provenientes de pesquisas que utilizam métodos combinados no mesmo estudo ou para sintetizar achados de uma mesma temática, de forma sistemática e rigorosa. Para isso, requer padrão de excelência quanto ao rigor metodológico para que seu produto possa trazer contribuições significativas para a ciência e para a prática clínica ${ }^{(24)}$.

Quanto à qualidade da evidência, foi verificado o predomínio de artigos classificados como NE 5. Para a aplicação da PBE este achado é preocupante, tendo em vista que as RS e metanálises são o padrão ouro na pesquisa, estando no topo da pirâmide. Este tipo de metodologia resultou do aumento da produção científica, tanto das pesquisas quantitativas quanto qualitativas, e tornou-se o fundamento do movimento da PBE em saúde ${ }^{(28-29)}$. O que se contrapõe com os resultados da presente revisão, na qual foram encontradas apenas duas $(9,5 \%)$ RS.

A problemática enfrentada se deve ao acúmulo de informações e pouco impacto dos resultados produzidos na prática assistencial, em detrimento de uma decisão exclusivamente regida pelas opiniões e experiência do profissional de saúde isoladamente ${ }^{(10,30)}$. O número reduzido de pesquisas realizadas por enfermeiros com melhores níveis de evidência, não significa que estes profissionais não estejam realizando intervenções; no entanto, traduz a fragilidade dos desenhos metodológicos que descrevem a utilização das intervenções realizadas no cenário brasileiro $^{(9)}$.

Considerando o contexto assistencial do país, a falta de aproximação dos profissionais à pesquisa, o baixo nível de participação dos enfermeiros em atividades científicas ${ }^{(1)}$ corrobora com os resultados apresentados nesse estudo, no qual, a maioria dos profissionais são os enfermeiros docentes que desenvolvem e utilizam a PBE.

Nesse aspecto, as mudanças identificadas nos últimos anos se devem à atuação dos enfermeiros docentes no processo de ensino-aprendizagem, desde a graduação, resultando em maior estímulo na busca de novos conhecimentos e da utilização de seus resultados para a melhoria da assistência. Assim, o maior número desses profissionais ingressantes em atividades de pós-graduação, como mestrado e doutorado e as diversas formas de discussão no tema, reforçam os resultados encontrados ${ }^{(10)}$.

Quanto à utilização da PBE na assistência, as dificuldades não se voltam apenas para a busca das evidências, mas também a utilização destas na sua realidade. A interligação entre a expertise 
do profissional e resultado em saúde necessita de ampla avaliação por parte de quem a executa. Não se pode apenas implementar uma evidência a qualquer tipo de situação, elas precisam ser similares ou próximas do contexto no qual a pesquisa foi realizada. Assim, na busca por uma efetividade do cuidado prestado, as intervenções de enfermagem precisam ser validadas para o contexto em que serão empregadas ${ }^{(1,5,30)}$.

Assim, diante dos desafios enfrentados para o desenvolvimento e implantação da PBE na enfermagem, estudos apontam que os enfermeiros demonstram elevado interesse e convicção em assumir que uma PBE conduziria a melhores cuidados e práticas clínicas, associando esta dimensão a um maior desenvolvimento profissional(29). Contudo demonstram mais segurança com alguém mais experiente ou com formação adequada, que auxilie no desenvolvimento e implementação da PBE na assistência ${ }^{(31)}$.

\section{CONCLUSÃO}

Os resultados obtidos com este estudo permitiram identificar a deficiência das pesquisas com melhores evidências científicas, para que seja ampliada a sua utilização na prática da enfermagem. Dentre as principais características dos artigos, tem-se as revisões como método mais utilizados nos estudos de enfermagem que utilizam a PBE, com nível de evidência 5. Com destaque as revisões integrativas e sistemáticas, direcionadas para a prática assistencial e de ensino, publicadas em periódicos nacionais nos últimos dez anos, sendo em maior número na região sudeste, por enfermeiros com atuação no ensino e titulação máxima de doutor.

Assim, observa-se que, embora a enfermagem tenha ampliado seus esforços para o avanço da EBE no cenário brasileiro, é necessária uma melhor organização com estudos de maior rigor metodológico, para garantir a tomada de decisões pelo enfermeiro em evidências científicas de alta qualidade e melhoria da assistência prestada.

Destaca-se também a importância de capacitação do enfermeiro assistencial na busca, desenvolvimento e utilização de pesquisas na prática profissional, a fim de transpor a dicotomia entre a pesquisa e o cuidar.

\section{REFERÊNCIAS}

1. Pedreira MLG. Enfermagem para a segurança do paciente. Acta Paul. Enferm. [Internet] 2009; 22(4) [acesso em 14 jul 2014]. Disponível: http://www.scielo. br/pdf/ape/v22n4/a01v22n4.pdf

2. Mendes KDS, Silveira RCCP, Galvão CM. Revisão integrativa: método de pesquisa para a incorporação de evidências na saúde e na enfermagem. Texto Contexto Enferm. [Internet] 2008; 17(4) [acesso em 16 jun 2014]. Disponível: http://dx.doi.org/10.1590/S010407072008000400018

3. Lacerda RA, Nunes BK, Batista Ade O, Egry EY, Graziano KU, Angelo M, et al. Evidence-based practices published in Brazil: identification and analysis of their types and methodological approches. Rev. Esc. Enferm. USP. [Internet] 2011; 45(3) [acesso em 14 jul 2014]. Disponível: http://dx.doi.org/10.1590/S008062342011000300033

4. Pompeo DA, Rossi LA, Galvão CM. Revisão integrativa: etapa inicial do processo de validação de diagnóstico de enfermagem. Acta Paul. Enferm. [Internet] 2009; 22(4) [acesso em 16 jun 2014]. Disponível: http://dx.doi. org/10.1590/S0103-21002009000400014

5. Pedrolo E, Danski MTR, Mingorance P, LazzariLSM; Méier MJ, Crozeta K. A prática baseada em evidências como ferramenta para prática profissional do enfermeiro. Cogitare enferm. [Internet] 2009; 14(4) [acesso em 14 jul 2014]. Disponível: http://ojs.c3sl.ufpr. br/ojs/index.php/cogitare/article/view/16396/10875

6. Santos CMC, Pimenta CAM, Nobre MRC. The PICO strategy for the research question construction and evidence search. Rev. Latino-Am. Enfermagem. [Internet] 2007; 15(3) [acesso em 16 jun 2014]. Disponível: http://www.scielo.br/pdf/rlae/v15n3/ v15n3a23.pdf

7. Cruz DAM, Pimenta CAM. Prática baseada em evidências, aplicada ao raciocínio diagnóstico. Rev. Latino-Am. Enfermagem. [Internet] 2005; 13(3) [acesso em 16 jun 2014]. Disponível: http://www.scielo.br/pdf/ rlae/v13n3/v13n3a17.pdf

8. Galvão CM, Sawada NO, Trevizan MA. Revisão sistemática: recurso que proporciona a incorporação das evidências na prática da enfermagem. Rev. LatinoAm. Enfermagem. 2004; 12(3) [acesso em 09 jun 2014]. Disponível:http://www.scielo.br/pdf/rlae/v12n3/ v12n3a14.pdf

9. Vasconcelos CTM, Damasceno MMC, Lima FET, Pinheiro AKB. Revisão integrativa das intervenções de enfermagem utilizadas para detecção precoce do câncer cérvico-uterino. Rev. Latino-Am. Enfermagem. [Internet] 2011; 19(2) [acesso em 14 jul 2014]. Disponível: http://www.scielo.br/pdf/rlae/v19n2/pt_28.pdf.

10. Galvão CM, Sawada NO. Prática baseada em evidências: estratégias para sua implementação na 
enfermagem. Rev. Bras. Enferm. [Internet] 2003; 56(1) [acesso em 16 jun 2014]. Disponível: http://www.scielo. br/pdf/reben/v56n1/a12v56n1.pdf

11. Souza MT, Silva MD, Carvalho R. Revisão integrativa: o que é e como fazer. Einstein. [Internet] 2010; 8(1) [acesso em 16 jun 2014]. Disponível: http://www. astresmetodologias.com/material/O_que_e_RIL.pdf

12. Souza V, Zeitoun SS, Barros ALBL. Débito cardíaco diminuído: revisão sistemática das características definidoras. Acta Paul. Enferm. [Internet] 2011; 24(1) [acesso em 16 jun 2014]. Disponível: http://www.scielo. br/pdf/ape/v24n1/v24n1a17.pdf

13. Oxford Centre for Evidence-Based Medicine. Levels of evidence [Internet] 2009 [acesso em 15 jul 2014]. Disponível: http://www.cebm.net/oxfordcentre-evidence-based-medicine-levels-evidencemarch-2009/

14. Azevedo EF, Barbosa LA, Cassiani SHB. Administração de antibióticos por via subcutânea: uma revisão integrativa da literatura. Acta Paul. Enferm. [Internet]2012; 25(5) [acesso em 16 jun 2014]. Disponível: http://www.scielo.br/pdf/ape/v25n5/26. pdf

15. Silva NCM, Chaves ECL, Carvalho EC, lunes DH. Instrumento para avaliação da integridade tissular dos pés de portadores de diabetes melittus. Acta Paul. Enferm. [Internet] 2013; 26(6) [acesso em 16 jun 2014]. Disponível: http://www.scielo.br/pdf/ape/v26n6/05. pdf

16. Silva JSF, Galvao TF, Pereira MG, Silva MT. Treatment of American tegumentaryleishmaniasis in special populations: a summary of evidence. Rev. Soc Bras Med Trop. [Internet] 2013; 46(6) [acesso em 16 jun 2014]. Disponível: http://www.ncbi.nlm.nih.gov/ pubmed/24474006

17. Rabelo ER, Aliti GB, Domingues FB, Assis MCS, Saffi MA, Linhares JC, et al. Enfermagem em cardiologia baseada em evidência. Rev HCPA. [Internet] 2007; 27(2) [acesso em 16 jun 2014]. Disponível: http://www. seer.ufrgs.br/hcpa/article/viewFile/2053/1163

18. Karino ME, Felli VEA. Enfermagem baseada em evidências: avanços e inovações em revisões sistemáticas. Ciênc Cuid Saúde. [Internet] 2012; 11(suppl) [acesso em 16 jun 2014]. Disponível: http:// periodicos.uem.br/ojs/index.php/CiencCuidSaude/ article/view/17048/pdf

19. Galvão CM, Sawada NO, Mendes IAC. A busca das melhores evidências. Rev. Esc. Enferm. USP. [Internet] 2003; 37(4) [acesso em 16 jun 2014]. Disponível: http:// www.scielo.br/pdf/reeusp/v37n4/05.pdf

20. Domenico EBL, Ide CAC. Enfermagem baseada em evidências: princípios e aplicabilidades. Rev. Latino-
Am. Enfermagem. [Internet] 2003; 11(1) [acesso em 16 jun 2014]. Disponível: http://www.revistas.usp.br/rlae/ article/viewFile/1743/1788

21. Santos DS, Carvalho EC. Intervenções de enfermagem para o cuidado de pacientes com artrite: revisão integrativa da literatura. Rev. Bras. Enferm. [Internet] 2012; 65(6) [acesso em 16 jun 2014]. Disponível:http://www.scielo.br/pdf/reben/v65n6/ a18v65n6.pdf

22. Mendes KDS, Galvão CM. Transplante de fígado: evidências para o cuidado de enfermagem. Rev. Latino-Am. Enfermagem. [Internet] 2008; 16(5) [acesso em 16 jun 2014]. Disponível: http://www.scielo.br/pdf/ rlae/v16n5/pt_19.pdf

23. Silva, SG, Nascimento ERP, Sales RK. Bundle de prevenção da pneumonia associada à ventilação mecânica: uma construção coletiva. Texto Contexto Enferm. [Internet] 2012; 21(4) [acesso em 16 jun 2014]. Disponível: http://www.scielo.br/pdf/tce/v21n4/14.pdf

24. Soares CB, Hoga LAK, Peduzzi M, Sangaleti C, YonekuraT, Silva DRAD. Revisão integrativa: conceitos e métodos utilizados na enfermagem. Rev. Esc. Enferm. USP. [Internet] 2014; 48(2) [acesso em 16 jun 2014]. Disponível: http://www.scielo.br/pdf/reeusp/v48n2/ pt_0080-6234-reeusp-48-02-335.pdf

25. Júnior FJ, Santos LC, Moura PV, Melo BM, Monteiro CF. Death and dying process: evidences from the literature of nursing. Rev. Bras. Enferm. [Internet] 2011; 64(6) [acesso em 16 jun 2014]. Disponível: http://www. scielo.br/pdf/reben/v64n6/v64n6a20.pdf

26. Toma TS, Rea MF. Benefits of breast feeding for maternal and child health: an essay on the scientific evidence.Cad. Saúde Publica. [Internet] 2008; 24Suppl 2 [acesso em 16 jun 2014]. Disponível: http://www. scielo.br/pdf/csp/v24s2/09.pdf

27. Oliveira MI, Camacho LA, Souza IE. Breastfeeding promotion, protection, and support in primary health care in the State of Rio de Janeiro, Brazil: a case of evidence-based public health policy.Cad. Saúde Publica. [Internet]2005; 21(6) [acesso em 16 jun 2014]. Disponível: http://www.scielosp.org/pdf/csp/v21n6/30. pdf

28. Matheus MCC. Metassíntese qualitativa: desenvolvimento e contribuições para a prática baseada em evidências. Acta Paul. Enferm. [Internet] 2009; 22(n.esp) [acesso em 16 jun 2014]. Disponível: http://www.redalyc.org/articulo.oa?id=307023853019

29. Castro AA. Revisão Sistemática e Meta-análise. [Internet] 2001; 3(1) [acesso 17 mai 2014]. Disponível: http://metodologia.org/wp-content/uploads/2010/08/ meta1.PDF

30. Fonseca C, Ramos A, Santos D, Gaspar L, Ferreira 
M. Prática de enfermagem baseada na evidência: o caso das feridas. Journal of Agindand Innovation. [Internet] 2012; 1(6) [acessoem 17 jul 2014]. Disponível: http://associacaoamigosdagrandeidade.com/revista/ volume1-edicao6-2012/pratica-de-enfermagem

31. Pereira RPG, Cardoso MJSPO, Martins MACSC. Atitudes e barreiras à prática de enfermagem baseada na evidência em contexto comunitário. Rev. Enf. Ref. [Internet] 2012; 3(7)[acesso em 17 jul 2014]. Disponível: http://www.scielo.mec.pt/pdf/ref/vserlIIn7/ serllIn7a06.pdf 